\title{
The effect of oscillating turbulent inflow on the shape of downstream turbulence
}

\author{
Young-Woo Yi*, Bhupendra Singh Chauhan** and Hee-Chang Lim**** \\ * School of Mechanical Engineering, Pusan National University, Busandaehak-ro 63beon-gil 2, Geumjeong-gu, Busan, Korea \\ ** Department of Mechanical Engineering, GLA University, Mathura, India \\ *** Corresponding Author : hclim@pusan.ac.kr
}

\begin{abstract}
Large Eddy Simulations (LES) has been widely applied and used in several decades to simulate a turbulent boundary layer in the numerical domain. In this study, we aimed to make a synthetic inflow generator (SIG) yielding an appropriate property of turbulent boundary layer in the inlet section and making quick development in the downstream of a three-dimensional domain. In order to achieve turbulent boundary layer quickly in a limited domain, the oscillating term was implemented in the well-defined boundary layer, which was expected to make faster convergence in the calculation. Cholesky decomposition was also applied to possess turbulent statistics such as the randomness and correlation of turbulent flow. In a result, the oscillating inflow did not show the faster convergence, but it indicated a possibility to improve statistical quantities in the downstream. In addition, regarding the mean flow characteristics were very close to the calculation without the oscillating flow. On the other hand, the turbulent statistics were improved depending on the oscillating magnitude.
\end{abstract}

Key words: CFD; Boundary layer flow; Correlation, LES; Cholesky decomposition.

\section{INTRODUCTION}

The atmospheric turbulence boundary layer has been applied in a variety of fields over a long period of time regarding the understanding of heat and mass transfer mechanisms [1-11]. Modelling the realistic atmospheric turbulent boundary layer is important design factor for the dynamic structures, such as wind turbines. It is ideal when the inlet conditions for the inlet condition of the flow simulation is numerically embodied atmospheric turbulent boundary layer having irregular and randomly fluctuated velocity components. However, most of the numerical simulation analysis the interaction between flow and wind turbine using uniform mean velocity or timely changed velocity condition $[12,13]$. Synthetic turbulent boundary layer mimicking the atmospheric turbulent boundary layer tends to have faster energy decay than the real one, so the synthetic boundary layer needs to have relatively bigger integral length scale than natural turbulent boundary layer.

Patel and Young [14] made the freestream flow having oscillating periodicity and convective velocity and did the experiment and numerical simulation to analyze the characteristics of the turbulent boundary layer. On the other hand, in this study, we wanted to find the difference of flow characteristics between the numerical simulation having nonoscillating inlet condition and having various oscillating inlet conditions.

Examining the turbulent boundary layer properties of the flow having time oscillating streamwise velocity inlet condition has two important findings. One is to embody the more realistic atmospheric turbulent boundary layer when finding the flow characteristics near the wind turbines. In previous researches, the inlet condition of the numerical 
flow simulation was set to measured or presumed data of the real cases to verify the real turbulent boundary layer experiment. In this case, much of the resources, such as time and disk space was considered to make the turbulent fully developed. In addition, when the inlet condition is established in numerous ways (integral length scales, magnitude of the stress, time-periodicity), the results themselves may have certain meanings.

This research aims to find the turbulent flow characteristics of the flow which the inlet condition made by time oscillating wave (sine, triangular, and trapezoidal) implemented SIG (Synthetic Inflow Generator). When the difference of the development of the turbulent caused by changing the shape of the oscillating wave which is implemented in SIG is found, it is possible to examine more accurate analysis of the fully developed turbulent flow.

\section{NUMERICAL METHOD}

\section{Governing Equation and Inlet Profiles}

In this study, first, in order to analyze the turbulent boundary layer between two parallel infinite plates, the incompressible and high resolution flow within the domain was considered. In addition, in order to create a turbulent boundary layer similar to the realistic one in the domain, the simulation of DNS (Direct Numerical Simulation), LES (Large Eddy Simulation) are prerequisite, but in terms of cost and practicality, the LES simulation was considered more suitable. The evaluation program used for flow analysis at the computational domain in this study was ANSYSFLUENT, a commercial thermal fluid program.

The governing equations in this study are spatially filtered incompressible Navier-Stokes equations as it can be seen at eqn. (1) and (2).

$$
\begin{gathered}
\frac{\partial \overline{u_{l}}}{\partial \bar{x}_{\imath}}=0 \\
\frac{\partial \overline{u_{l}}}{\partial t}+\bar{u}_{\jmath} \frac{\partial \overline{u_{l}}}{\partial \overline{x_{\jmath}}}=-\frac{1}{\rho} \frac{\partial \bar{p}}{\partial \bar{x}_{\imath}}+2 v \frac{\partial}{\partial \bar{x}_{\jmath}} \overline{S_{l \jmath}}-\frac{\partial \tau_{i j}^{r}}{\partial x_{j}}
\end{gathered}
$$

To make the synthetic turbulent boundary layer flow used at the inlet of the domain, the actual turbulent characteristics should be considered. Detailed information related to this study is written at reference paper [15]. The brief summary is written here.

Like eqn. (3), the instantaneous velocity in the flow domain can be separated to the mean and fluctuating component by Reynolds decomposition theory.

$$
u_{i}=\bar{u}_{\imath}+\alpha_{i j} \psi_{j}
$$

where is the mean velocity, is the amplitude tensor, and is fluctuating components having 0 mean value and 1 variance. In this study, the amplitude tensor is used which is suggested by Lund et. al [16] as it can be seen at eqn. (4). is the Reynolds stress tensor acquired from experiment or numerical simulation. 


$$
\alpha_{i j}=\left(\begin{array}{ccc}
\sqrt{R_{11}} & 0 & 0 \\
R_{21} / \alpha_{11} & \sqrt{R_{22}-\alpha_{21}^{2}} & 0 \\
R_{31} / \alpha_{11} & \left(R_{32}-\alpha_{21} \alpha_{31} / \alpha_{22}\right) & \sqrt{R_{33}-\alpha_{31}^{2}-\alpha_{32}^{2}}
\end{array}\right)
$$

\section{Boundary Conditions}

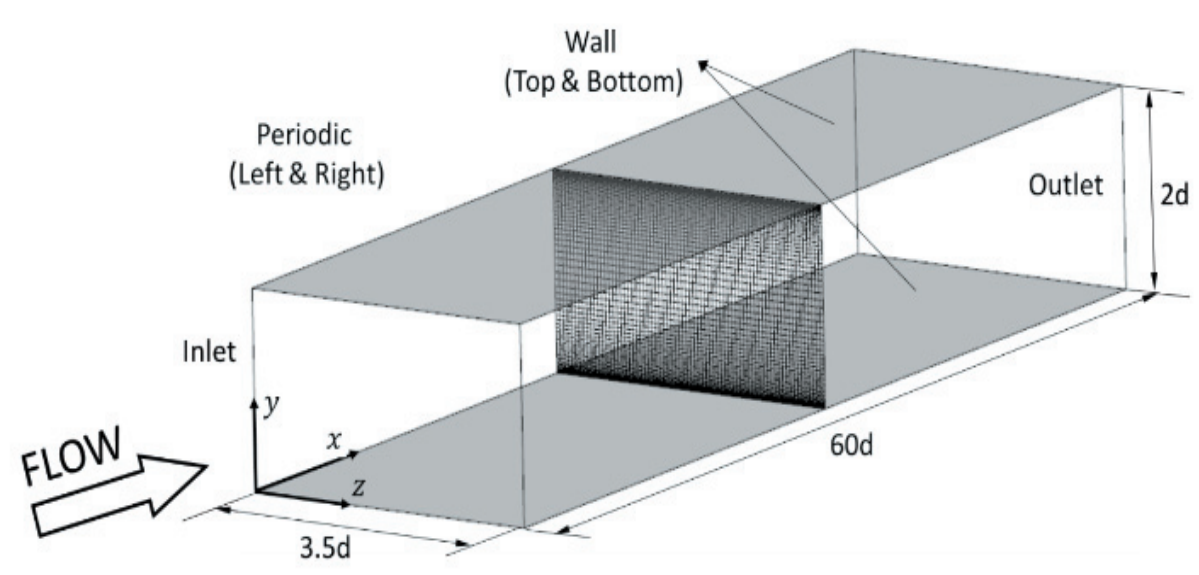

Figure 1. Schematics of computational channel domain

In this study, the hexahedral grid system was used because it could do more efficient turbulent flow simulation. To get better resolution of vortex eddy near the flat plate made by the development of the turbulent boundary layer, the density of grid near the plate was relatively higher comparing to the other region. The turbulent Reynolds number $\left(R e_{\tau}=123.7\right)$ was drawn by friction velocity $\left(u_{\tau}\right)$ at the flat plate and a half height of the channel $(d)$. No-slip condition was used at the top and bottom flat plates and periodic condition was used at the side of the flow domain. In addition, velocity-inlet condition was used at inlet and zero-gradient condition at outlet because the flow properties should not be diffused. The size of the flow domain was $60 \mathrm{~d} \times 2 \mathrm{~d} \times 3.5 \mathrm{~d}$, which stands for streamwise, vertical, and spanwise direction, respectively. To make the flow simulation can be converged, the number of grid reached nearly 2,500,000 and schematics of the flow domain can be seen at Fig. 1. The grid is made by the consideration of the convergence based on LES, so near the wall was less than 1. Furthermore, the non-dimensional grid size at streamwise and spanwise direction was set to $\Delta \mathrm{x}^{+}=15, \Delta \mathrm{z}^{+}=7.5$.

\section{TURBULENT BOUNDARY LAYERS HAVING OSCILLATING FLOW}

Like eqn. (5), the oscillating mean flow velocity can be separated to the mean component and the oscillating component as follows,

$$
\mathrm{U}(x, t)=U_{0}\left\{1+N \sin \left(\omega\left(t-\frac{x}{Q}\right)\right)\right\}
$$


where $U$ is the flow velocity, which is the function of flow direction and time, $U_{0}$ is the mean velocity, and $N$ is the amplitude of the oscillation non-dimensionalized by mean velocity. In addition, $\omega$ is the frequency(rad) and $Q$ is the travelling wave convective velocity. In this study, the inlet condition is assumed to be similar to the purely time dependent freestream oscillation flow suggested by Patel and Young [14], as shown in eqn. (6).

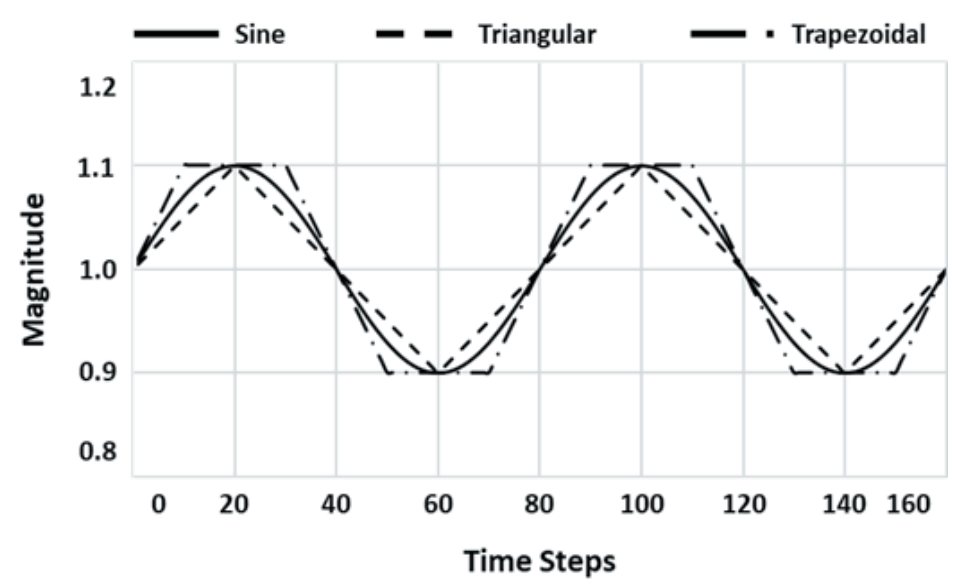

Figure 2. Variation of oscillating function in inlet conditions

$$
\mathrm{U}(y, z, t)=U_{0}(y, z)\{1+N \times A(T(t))\}
$$

where $U$ is the flow velocity, which is the function of vertical direction $(y)$, spanwise direction $(z)$, and $\operatorname{time}(t) . U_{0}$ is mean velocity in terms of time. $N$ is the amplitude of the oscillation function $A(T(t))$ and $T(t)$ is the periodic parameter of the oscillation function as shown in Fig.2.

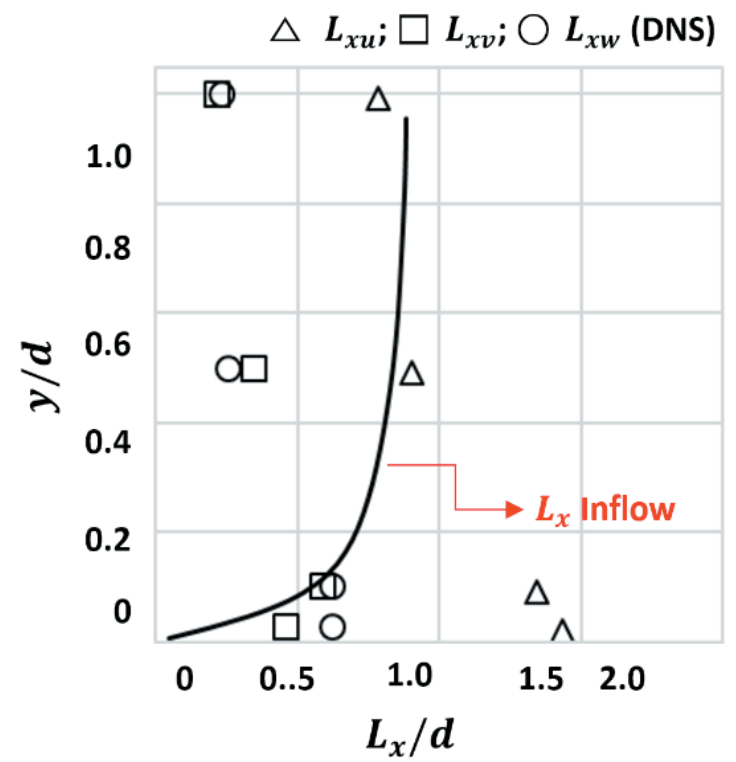

Figure 3. Profiles of integral lengthscales used in the inlet section of the domain 


\section{RESULTS AND DISCUSSION}

\section{Calculation of Inlet Velocity Profiles}

Inlet velocity profiles of the flow domain are the crucial foundation of this study. The correlation based on the lengthscales of the turbulent boundary layer flow should be applied at the inlet velocity profiles. As described earlier, this study aims to make proper turbulent boundary layer properties in flow domain using the results of DNS [17].

Figure 3 shows the comparison between the integral lengthscales of the DNS at the normal direction to the wall and those at inlet velocity profiles in this study. The subscript $u, v$, and $w$ are the velocity components at streamwise, normal to the wall, and spanwise direction each.

The integral lengthscles $L_{i}$ can be defined like eqn. (7).

$$
L_{i}=\int_{i}^{\infty} R\left(x_{i}\right) d x_{i}, i=1,2,3
$$

The solid line in Fig. 3 represents the magnitude of integral lengthscales of inlet streamwise velocity. Based on Taylor's hypothesis, lengthscales of streamwise velocity can be defined by the product of mean velocity $\left(\bar{u}_{\imath}\right)$ and Lagrangian time scale $(T)$ when turbulent intensity is relatively low. Therefore, $L_{x}=T \times \bar{u}$ can be applicable [18].

In this study, the turbulent intensity is less than 0.3, so the Taylor's hypothesis can be applied in the whole flow domain. The lengthscales of streamwise velocity in this study is proportional to mean velocity because $T$ is constant. However, the lengthscales derived by DNS data have bigger value when the results are close to the wall. It seems that to make the lengthscales similar to the DNS' results, the 3D domain of the flow domain should be considered when generating fluctuating components $\left(\psi_{i}\right)$.

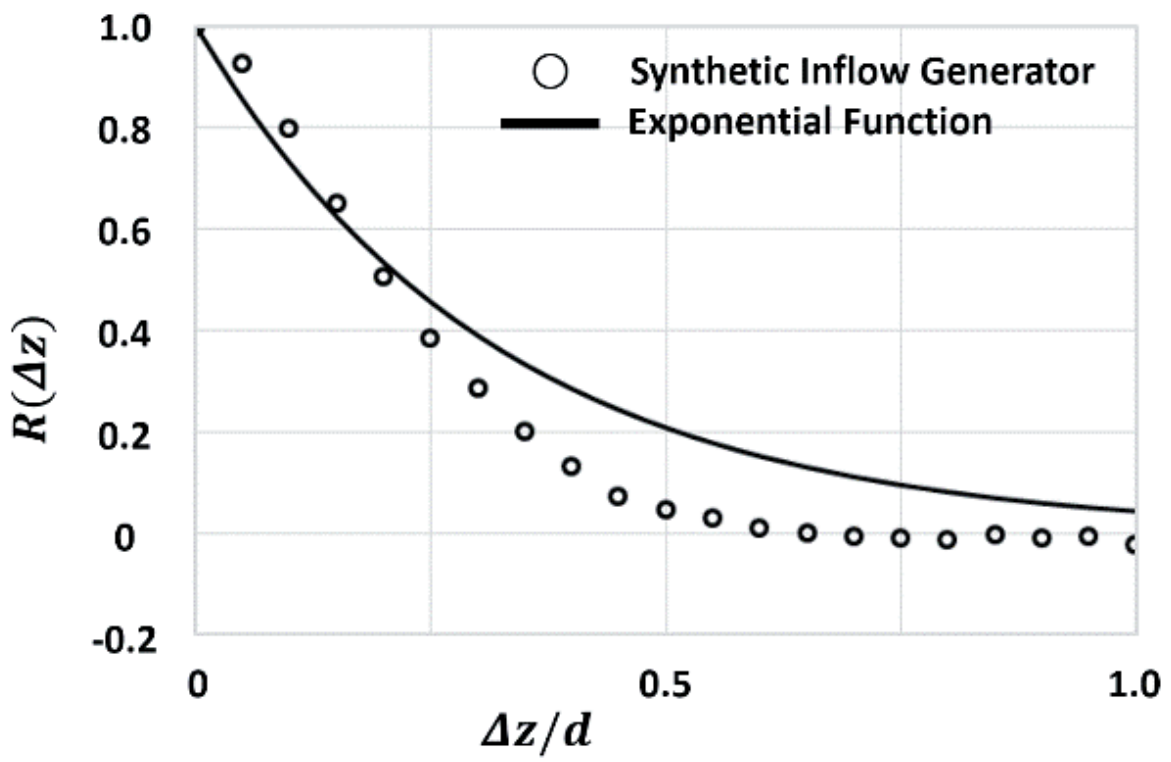

Figure 4. Spatial correlation function of input turbulent data from synthetic inflow generator and exponential function 
Figure 4. shows the comparison between the correlation of the time and spatial filtered random number made by random function and the correlation of the turbulent flow. Usually, the characteristics of turbulent flow can be represented in the form of exponentially decayed function. So the comparison of correlation should be needed whether the SIG (Synthetic Inflow Generator) is suitable. In Fig. 4, the correlation of the turbulent flow using SIG decays exponentially.

\section{TURBULENT FLOW CHARACTERISTICS}

In this study, four different types of turbulent boundary layer data are made by SIG and used at inlet velocity profiles in flow domain. One is the most common, non-oscillatory SIG (hereafter, Inflow Normal). The other three SIG have oscillating components (Sine, Triangular, and Trapezoidal function).

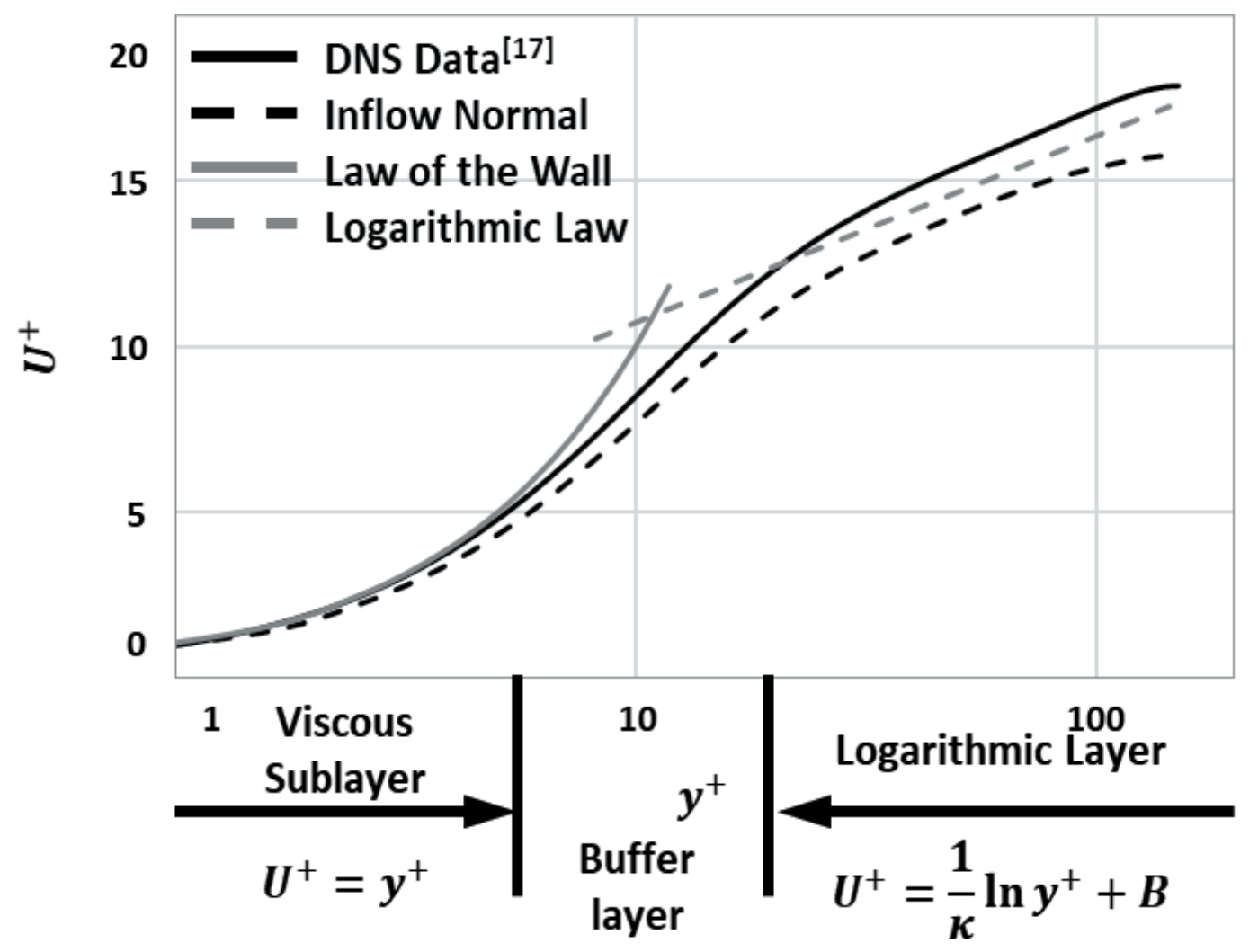

Figure 5. Comparison of among DNS data (Iwamoto, 2002), Synthetic Inflow data, and theoretical results

Figure 5 shows the comparison between Inflow Normal inlet velocity profiles and DNS data. As shown in the figure, the DNS data where $y^{+}$is small (close to the wall) is closer to the law of the wall than the profiles of Inflow Normal. However, as $y^{+}$reaches at the range of logarithmic region, both profiles are similar to the theory. 


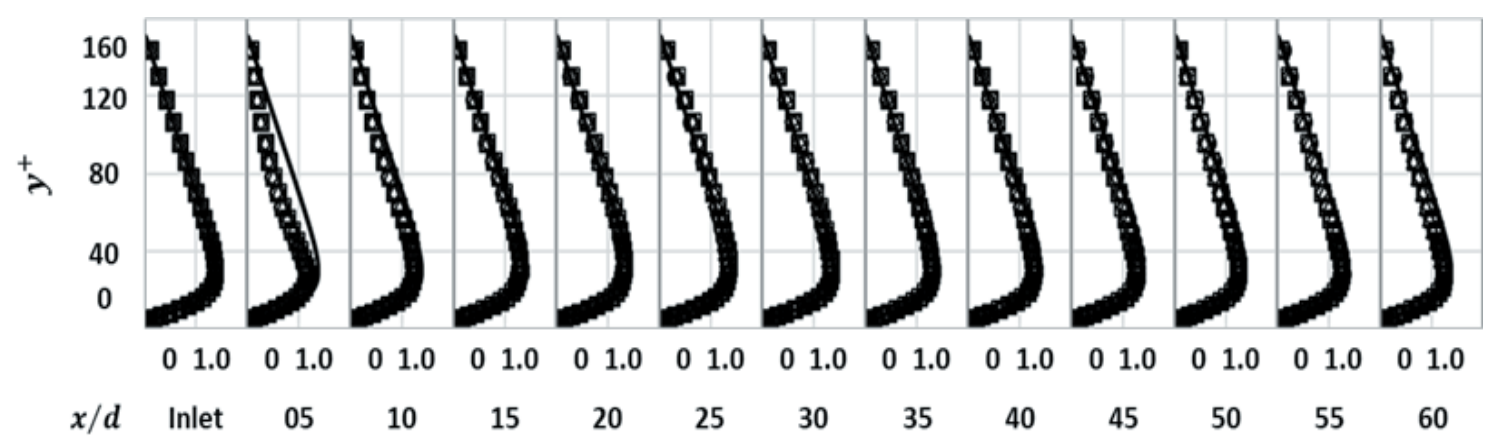

Figure 6 Comparison of $U^{+}$among DNS data [17] and the cases of synthetic inflow data(Black line : DNS data [15], $\bigcirc$ : Inflow Normal, $\square$ : Inflow Sine, $\diamond$ : Inflow Triangular, $\triangle$ : Inflow Trapezoidal)

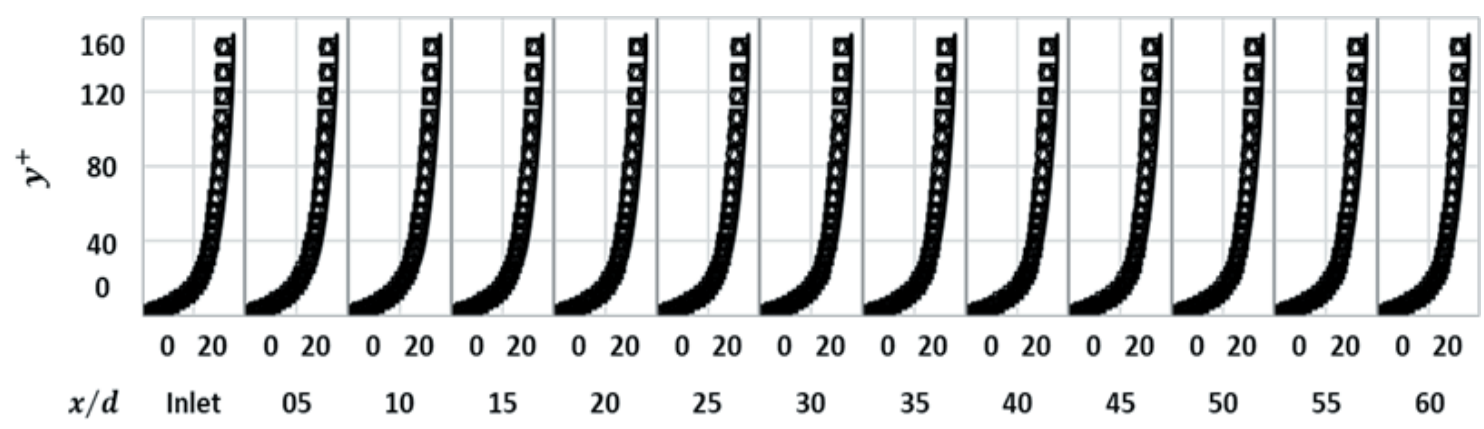

Figure 7 Comparison of $\left\langle-u^{\prime} v^{\prime}\right\rangle^{+}$among DNS data [17] and the cases of synthetic inflow data(Black line : DNS data [15], $\bigcirc$ : Inflow Normal, $\square$ : Inflow Sine, $\diamond$ : Inflow Triangular, $\triangle$ : Inflow Trapezoidal)

Figure 6 and 7 are the comparison between $\square$ the DNS data and simulation results to find the place where the four turbulent boundary layer flows are fully developed. In Fig. 6, in terms of $u^{+}$, the comparison of four different SIG results shows no difference compared to the DNS data. In Fig. 7, $\left\langle-u^{\prime} v^{\prime}\right\rangle^{+}$is used for an indicator to find the location of fully development of turbulent boundary layer. As shown in those figures, $\left\langle-u^{\prime} v^{\prime}\right\rangle^{+}$is more suitable indicator than for turbulent flow analysis.

Figure 7 shows the approximate location where the turbulent flow is fully developed, so the further process like Eqn. (8) is needed.

Figure 8 shows the behavior of Error $\left(\left\langle-u^{\prime} v^{\prime}\right\rangle^{+}\right)$in terms of streamwise location. Most of inflow models have the biggest value at $a x d t=5$. Before $x d$ reaches Error $\left(\left\langle-u^{\prime} v^{\prime}\right\rangle^{+}\right) 15$ to 20 , the decreases, and after reaching $x d d=30$ to 40 , the value is gradually increased. So where $x d d=20$, the turbulent flows are fully developed. It seems that these behaviors are caused by the dispersion of the turbulent flow. Inflow models using oscillatory functions have bigger range of differences of after the turbulent flows are fully developed. The instability of the flow increases when the oscillatory functions are added to the SIG. 


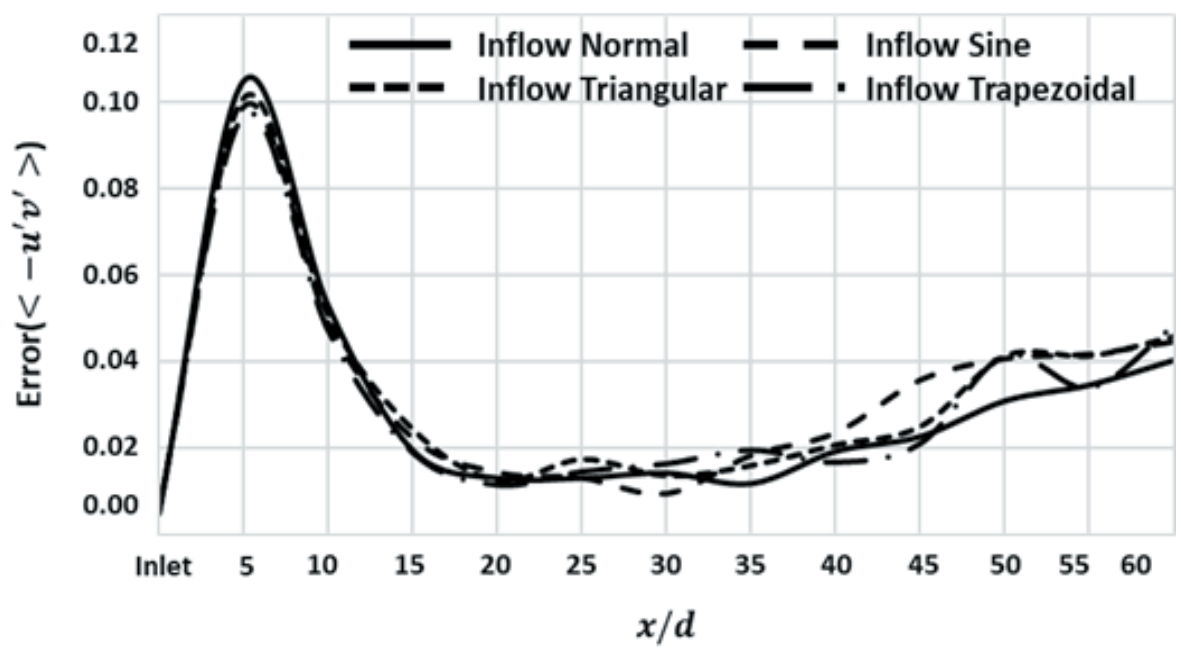

Figure 8. Error $\left(\left\langle-u^{\prime} v^{\prime}\right\rangle^{+}\right)$tendency graph in change of streamwise location

$$
\text { Error }=\frac{\sum_{1}^{j_{\max }} A b s\left(\left\langle-u^{\prime} v^{\prime}\right\rangle_{j, D N S}^{+}-\left\langle-u^{\prime} v^{\prime}\right\rangle_{j, C h}^{+}\right)}{j_{\max }}
$$

It is important to see the variation of turbulent boundary layer profiles when the oscillatory functions are added to the SIG. As shown in Fig. 9, the additional oscillatory functions can make no certain changes at $u^{+}$and other Reynolds stresses $\left(\left\langle v^{\prime} v^{\prime}\right\rangle^{+},\left\langle w^{\prime} w^{\prime}\right\rangle^{+},\left\langle-u^{\prime} v^{\prime}\right\rangle^{+}\right)$. On the other hand, axial stresses $\left(\left\langle u^{\prime} u^{\prime}\right\rangle^{+}\right)$from the oscillatory function are bigger at $y^{+}$is less than 5 and bigger than 40 .

Wall shear stress comparison between the Inflow Normal and the results of other Inflow models can be seen at Fig. 10. In all cases, wall shear stresses near the inlet are big, and become constant as the $x d$ increases. The magnitude of the wall shear stresses of oscillatory Inflow models are slightly bigger than that of Inflow Normal.

Turbulent boundary layer flow is typically random and irregular. Modelling real atmospheric turbulent boundary layer and acquiring proper turbulent properties is not only important design base for the dynamic structures such as wind turbine but also crucial factor for success at the wind energy business. 

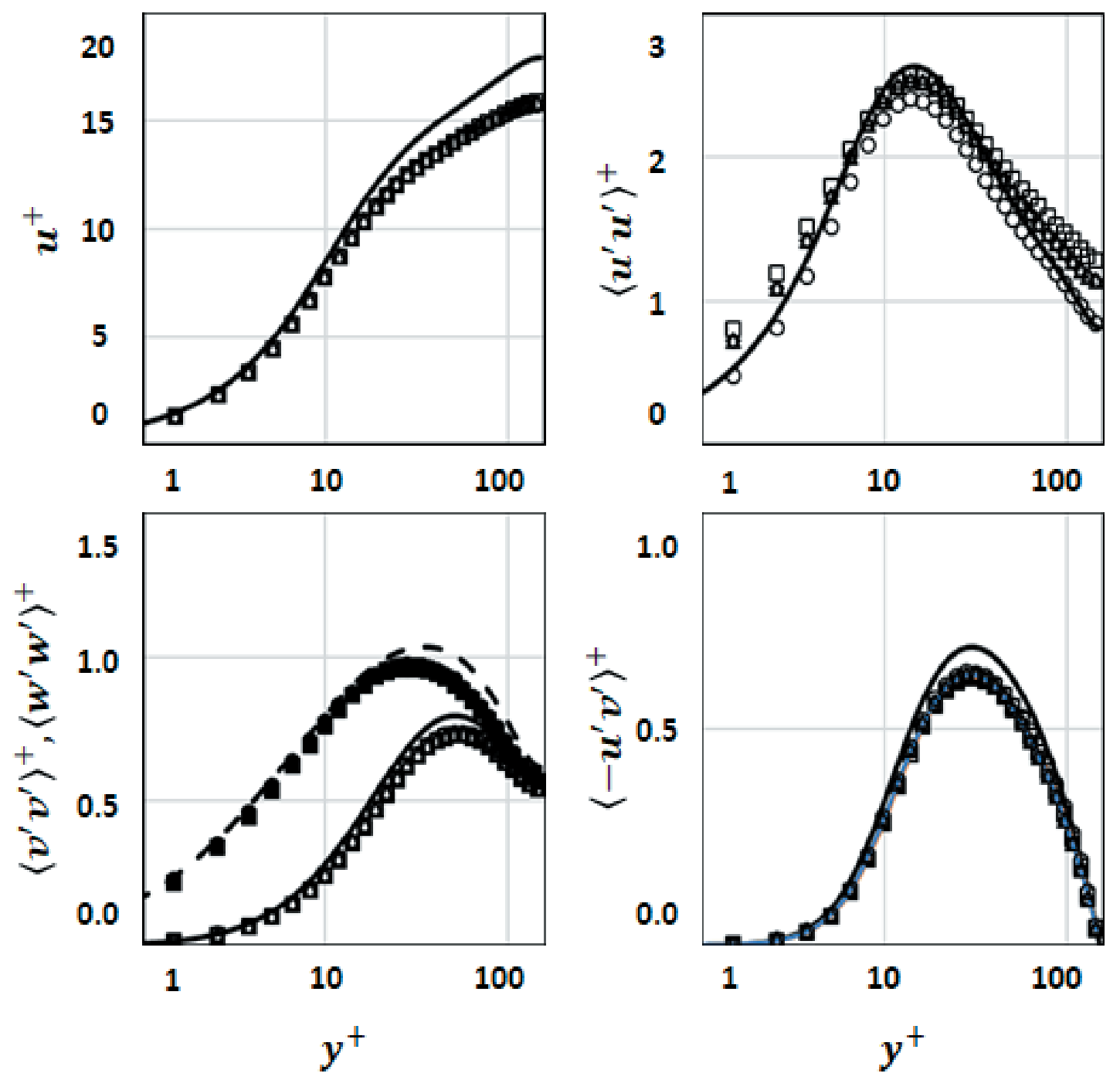

Figure 9. Comparison of turbulent data among DNS data [17] and four cases of synthetic inflow turbulent data at $x / d=50$ (Black line : DNS data [17], $\bigcirc:$ Inflow Normal, $\square:$ Inflow Sine, $\diamond:$ Inflow Triangular, $\triangle$ : Inflow Trapezoidal)

\section{CONCLUSIONS}

In this study, synthetic inflow generator is used to make the flow downstream fully developed. SIGs having oscillatory functions are used to find the change of response characteristics of the flow when the lengthscles of the inlet velocity profiles are changed. 


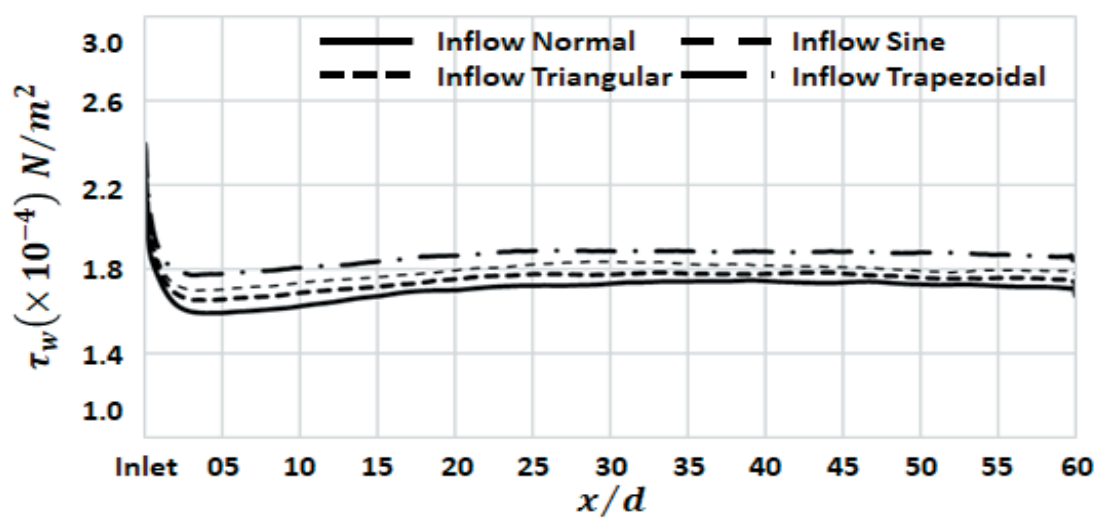

Figure 10. Comparison of shear stress $\left(\tau_{w}\right)$ among four cases of synthetic inflow data against $x / d$

The results can be summarized.

1. Synthetic method is used to model the atmospheric turbulent boundary layer, and turbulent

2. properties are well matched with the DNS data for the wind turbine business.

3. SIGs having oscillatory functions also can be fully developed in the flow domain.

4. $\left\langle-u^{\prime} v^{\prime}\right\rangle^{+}$is useful indicator for testing a development of turbulent boundary layer flow.

5. The instability of the flow can be increased when the inlet profiles have oscillatory functions.

In this study, there have been many things to improve to model realistic turbulent boundary layer. At first, the effect of period and magnitude of time oscillating flow are still questionable. The effect of the wall temperature and temperature of other boundary conditions also can be interesting topics for modelling real atmospheric turbulent boundary layer. In addition, further research is needed about to compare with the experiment and numerical simulation of bluff body cases which turbulent results of this study are used as inlet condition.

\section{ACKNOWLEDGEMENT}

This work was supported by 'Human Resources Program in Energy Technology' of the Korea Institute of Energy Technology Evaluation and Planning (KETEP), granted financial resource from the Ministry of Trade, Industry \& Energy, Republic of Korea (no. 20184030202200). In addition, this work was supported by the National Research Foundation of Korea (NRF) grant funded by the Korea government (MSIP) (no. 2019R1I1A3A01058576). This work was also supported by the National Supercomputing Center with supercomputing resources including technical support (KSC-2020-INO-0025).

\section{REFERENCES}

Kovasznay, L. S., 1970, The Turbulent Boundary Layer. Annual Review of Fluid Mechanics 2 : 95 112.

Willmarth, W. W., 1975. Pressure Fluctuations Beneath Turbulent Boundary Layers. Annual Review of Fluid Mechanics. $7:$ 13 38.

Kline, S. J., Reynolds, W. C., Schraub, F. A., and Runstadler, P. W., 1979. The structure of turbulent boundary layers. Journal of Fluid Mechanics. 30 (4) : 741 773. 
Sreenivasan, K. R., 1989. The turbulent boundary layer. Frontiers in experimental fluid mechanics. Springer, Berlin, Heidelberg : 159 209.

Kline, S. J. and Robinson, S. K., 1990. Quasi-coherent structures in the turbulent boundary layer. I-Status report on a community-wide summary of the data. Near-wall turbulence. : 200 217.

Lim, H. C., 2007. Flow Structure on the Roof of a Cube Placed in a Turbulent Boundary Layer. J. Wind Eng. Ins. Korea. 11 (2) : 153 159.

Jeong, T. Y. and Lim, H. C., 2010. The Effects of Atmospheric Stability on Flow Structure around a Complex Terrain. J. Wind Eng. Ins. Korea. 14 (1) : 47 54.

Lee, Y. T. and Lim, H. C., 2011. Study on the Surface Pressure Variation around 2 and 3-Dimensional Trench Cavities. J. Wind Eng. Ins. Korea. 15 (4) : 117 122.

Yang, H. B. and Lim, H. C., 2012. Effect of Azimuth angle on Wind Load around Serially Aligned Buildings. J. Wind Eng. Ins. Korea. 16 (3) : 101 107.

Liu, Y., Wang, L., Zhu, Z., 2016. Experimental and numerical studies on the effect of inlet pressure on cavitating flows in rotor pumps. Journal of Engineering Research. 4 (2) : 1-21.

Yu, H., Duan, S., Sun, P., 2015. Comparative analysis between natural gas/diesel (dual fuel) and pure diesel on the marine diesel engine. Journal of Engineering Research. 3 (4) : 1-15.

Kim, M. O., Kim, B. S., Mo, J. H., and Lee, Y. H., 2010. Aerodynanamic design and performance computational of a 5kW HAWT rotor blades. Journal of Wind Energy. 1 (2) : 47 52.

Nam, S. H., Choi, N. J., Jeong, J. H., and Kim, B. S., 2010. 6MW Class Wind Turbine Blade Design and CFD Computational by Using XD-Blade. Journal of Wind Energy. 1 (1) : 6 11.

Patel, M. H. and Young, A. D., 1977. On turbulent boundary layers in oscillatory flow. Proceedings of the Royal Society of London. A. Mathematical and Physical Sciences. 353 (1672) : 121 144.

Lee, Y. T. and Lim, H. C., 2017. Effect of Inflow Length Scales on the Generation of Turbulent Boundary Layer Flow. J. Wind Eng. Ins. Korea. 21 (4) : 171 178.

Lund, T. S., Wu, X., and Squires, K. D., 1998. Generation of turbulent inflow data for spatially-developing boundary layer simulations. Journal of Computational Physics. 140 (2) : 233 258.

Iwamoto, K., Suzuki Y., and Kasagi, N., 2002. Database of Fully Developed Channel Flow-THTLAB Internal Report No. ILR-0201, Rapport technique. THTLAB, Dept. of Mech. Eng., The Univ. of Tokyo (CH12_PG.WL7).

Taylor, G. I., 1938. The spectrum of turbulence. Proceedings of the Royal of the Royal Society of London. Series A-Mathematical and Physical Sciences. 164 (919) : 476 490. 